\title{
Mott-Driven BEC-BCS Crossover in a Doped Spin Liquid Candidate $\kappa-(\text { BEDT-TTF })_{4} \mathrm{Hg}_{2.89} \mathrm{Br}_{8}$
}

\author{
Y. Suzuki®, ${ }^{1, \dagger}$ K. Wakamatsu $\odot,{ }^{1, \dagger}$ J. Ibuka $\odot,{ }^{1}$ H. Oike $\odot,{ }^{1}$ T. Fujii, ${ }^{2}$ K. Miyagawa, ${ }^{1}$ H. Taniguchi $\odot,{ }^{3}$ and K. Kanoda $\odot^{1, *}$ \\ ${ }^{1}$ Department of Applied Physics, University of Tokyo, Bunkyo-ku, Tokyo, 113-8656, Japan \\ ${ }^{2}$ Cryogenic Research Center, University of Tokyo, Bunkyo-ku, Tokyo, 113-0032, Japan \\ ${ }^{3}$ Graduate School of Science and Engineering, Saitama University, Saitama 338-8570, Japan
}

(Received 22 July 2021; revised 12 October 2021; accepted 12 November 2021; published 25 January 2022)

\begin{abstract}
The pairing of interacting fermions leading to superfluidity has two limiting regimes: the BardeenCooper-Schrieffer (BCS) scheme for weakly interacting degenerate fermions and the Bose-Einstein condensation (BEC) of bosonic pairs of strongly interacting fermions. While the superconductivity that emerges in most metallic systems is the BCS-like electron pairing, strongly correlated electrons with poor Fermi liquidity can condense into the unconventional BEC-like pairs. Quantum spin liquids harbor extraordinary spin correlation free from order, and the superconductivity that possibly emerges by carrier doping of the spin liquids is expected to have a peculiar pairing nature. The present study experimentally explores the nature of the pairing condensate in a doped spin liquid candidate material and under varying pressure, which changes the electron-electron Coulombic interactions across the Mott critical value in the system. The transport measurements reveal that the superconductivity at low pressures is a BEC-like condensate from a non-Fermi liquid and crosses over to a BCS-like condensate from a Fermi liquid at high pressures. The Nernst-effect measurements distinctively illustrate the two regimes of the pairing in terms of its robustness to the magnetic field. The present Mott tuning of the BEC-BCS crossover can be compared to the Feshbach tuning of the BEC-BCS crossover of fermionic cold atoms.
\end{abstract}

DOI: 10.1103/PhysRevX.12.011016

\section{INTRODUCTION}

Strong interactions between itinerant electrons in solids cause peculiar correlations or organizations among the electrons beyond the Fermi-liquid (FL) framework [1]. Superconductivity emerging in these circumstances can be outside the celebrated Bardeen-Cooper-Schrieffer (BCS) framework, which assumes an instability of Fermi surfaces formed by degenerate fermions with well-defined momenta [2], in that the strong correlation of electrons makes their momenta ill defined and the electron pairing in momentum space, the central idea of BCS theory, is less pertinent. In this case, superconductivity is of the Bose-Einstein condensation (BEC) type, as the size of Cooper pairs is as small as their mean distance, or equivalently, the interaction energy is comparable to the Fermi energy. Several unconventional superconductors have been discussed in the

\footnotetext{
${ }^{*}$ Corresponding author. kanoda@ap.t.u-tokyo.ac.jp

${ }^{\dagger}$ These authors contributed equally to this work.

Published by the American Physical Society under the terms of the Creative Commons Attribution 4.0 International license. Further distribution of this work must maintain attribution to the author(s) and the published article's title, journal citation, and DOI.
}

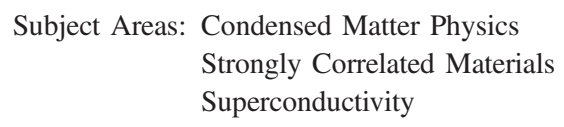

framework of a BEC scenario [3-7]. More broadly, the superfluidity of cold fermionic atoms is known to be controllable between the BEC and BCS regimes by the magnetic-field tuning of the interatomic interaction through the Feshbach resonance $[8,9]$. The relation between the pairing nature and the interparticle correlation is a seminal issue in the instability of interacting fermions at large $[10,11]$. Thus, it is expected to identify interacting systems having novel types of correlation and explore the nature of the pairing condensate therein under variation in the interaction in a controllable manner.

Quantum spin liquids emerging on geometrically frustrated lattices are argued to possess peculiar spin correlations possibly linked to fractionalization, entanglement, and/or chirality in magnetic excitations [12-14]. The superconductivity possibly brought about by doping against such an anomalous magnetic state [15-23] is expected to have unconventional pairing nature out of the BCS framework; however, it has yet to be explored experimentally. In the present study, we address this issue by working with a doped spin liquid candidate under pressure variation, and reveal that superconductivity in this system exhibits a novel-type Mottness-controlled BEC-BCS crossover.

The material studied is a layered organic superconductor, $\kappa$-(BEDT-TTF $)_{4} \mathrm{Hg}_{2.89} \mathrm{Br}_{8} \quad(\kappa-\mathrm{HgBr}$ hereafter $)$, in which 
(a)

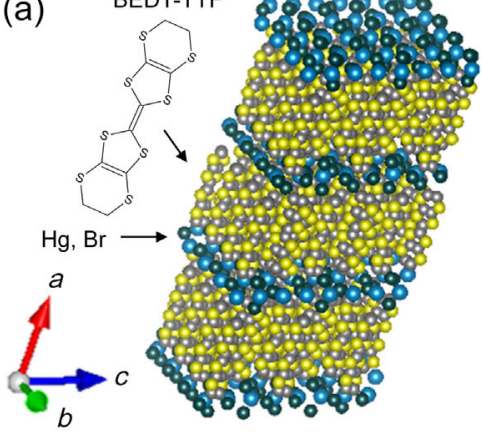

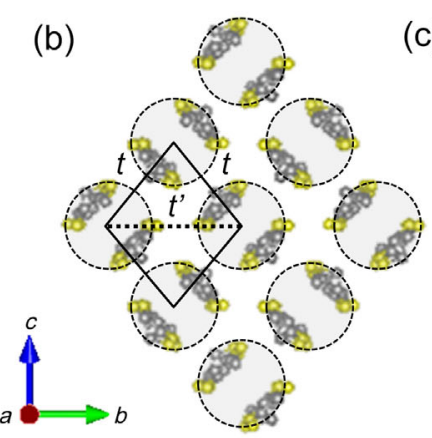

FIG. 1. Structure and phase diagram of $\kappa$-(BEDT-TTF $)_{4} \mathrm{Hg}_{2.89} \mathrm{Br}_{8}$. (a) Layered structure consisting of BEDT-TTF conducting layers and $\mathrm{Hg}_{2.89} \mathrm{Br}_{8}$ insulating layers. BEDT-TTF denotes bis(ethylenedithio)tetrathiafulvalene. The nonstoichiometry in the $\mathrm{Hg}$ composition comes from the fixed incommensurability between the $\mathrm{Hg}$ and BEDT-TTF lattices originating from material chemistry, not from extrinsic defects. (b) In-plane structure of the conducting layers. BEDT-TTF molecules form dimers, which constitute a nearly isotropic isosceles triangular lattice with the anisotropy of transfer integrals $t^{\prime} / t$ close to unity. (c) Pressure-temperature phase diagrams of $\kappa$-(BEDT-TTF $)_{4} \mathrm{Hg}_{2.89} \mathrm{Br}_{8}$ and a quantum spin liquid candidate $\kappa$-(BEDT-TTF $)_{2} \mathrm{Cu}_{2}(\mathrm{CN})_{3}$. The red area indicates the superconducting phase. The blue area in $\kappa$-(BEDT-TTF $)_{4} \mathrm{Hg}_{2.89} \mathrm{Br}_{8}$ depicts the non-Fermi-liquid region [corresponding to the yellow region in Fig. 2(c)]. The purple area in $\kappa$-(BEDT-TTF $)_{2} \mathrm{Cu}_{2}(\mathrm{CN})_{3}$ depicts the Mott-insulating spin liquid phase [42,43]. $T_{c}$ in $\kappa$-(BEDT-TTF $)_{2} \mathrm{Cu}_{2}(\mathrm{CN})_{3}$ is taken from Ref. [42], and $T_{c}$ in $\kappa$-(BEDT-TTF $)_{4} \mathrm{Hg}_{2.89} \mathrm{Br}_{8}$ corresponds to the midpoint of the resistive transition observed in this study. MI, QSL, NFL, and SC represent the Mott insulator, quantum spin liquid, non-Fermi liquid, and superconductivity, respectively. The white broken line indicates the location of the so-called $6 \mathrm{~K}$ anomaly observed in $\kappa$-(BEDT-TTF) ${ }_{2} \mathrm{Cu}_{2}(\mathrm{CN})_{3}[12,41,42]$, a possible indication of an instability of QSL.

dimers of BEDT-TTF molecules constitute a nearly perfect isotropic triangular lattice, separated by insulating $\mathrm{Hg}_{2.89} \mathrm{Br}_{8}$ layers [Figs. 1(a) and 1(b)] [24,25]. The nonstoichiometry of $\mathrm{Hg}$ arises from its sublattice incommensurate with the host lattice formed by BEDT-TTF and Br, and the composition of $\mathrm{Hg}$ is reproducibly given by the mismatch between the two lattices precisely determined by $\mathrm{x}$-ray diffraction $[24,25]$. The triangular lattice structure is almost identical to that of the Mott-insulating spin liquid candidate $\kappa$-(BEDT-TTF $)_{2} \mathrm{Cu}_{2}(\mathrm{CN})_{3} \quad\left[\kappa-\mathrm{Cu}_{2}(\mathrm{CN})_{3}\right.$ hereafter], in which the spin-1/2 localized on each BEDT-TTF dimer interacts with its neighbors through an antiferromagnetic exchange energy $J$ of $250 \mathrm{~K}$ but does not order down to $30 \mathrm{mK}$ [34-36]. A distinction of $\kappa-\mathrm{HgBr}$ from $\kappa-\mathrm{Cu}_{2}(\mathrm{CN})_{3}$ is that the nonstoichiometric $\mathrm{Hg}$ ions give $11 \%$ hole doping to the Mott insulator, resulting in a metallic state $[25,37,38]$. Surprisingly, the $J$-scaled spin susceptibility, namely, $J \chi_{\text {spin }}$, versus $T / J \chi_{\text {spin }}[J=140$ and $250 \mathrm{~K}$ for $\kappa-\mathrm{HgBr}$ and $\kappa-\mathrm{Cu}_{2}(\mathrm{CN})_{3}$, respectively], nearly coincide between $\kappa-\mathrm{HgBr}$ and $\kappa-\mathrm{Cu}_{2}(\mathrm{CN})_{3}$, and are well reproduced by the triangular-lattice antiferromagnetic Heisenberg model, suggesting that $\kappa-\mathrm{HgBr}$ hosts a quantum spin liquid (QSL) in the spin sector while remaining metallic in the charge sector [39]. Thus, $\kappa-\mathrm{HgBr}$ is a possible realization of the long sought-after doped QSL [14]. According to the transport study $[37,40]$, the metallic state at low pressures is a non-Fermi liquid (NFL), and the doped holes, not particles of the entire band, carry mobile charges. Superconductivity in such a doped QSL candidate [24,37], possibly appearing in place of the low-temperature instability of the QSL [41], is of profound interest [15-23].
Pressure is an effective control parameter of electronelectron interactions in molecular materials, which are highly compressible in general [44]. The transport measurements indicate that when $\kappa-\mathrm{HgBr}$ is pressurized in excess of $5 \mathrm{kbar}$, all band particles become mobile [37], and concomitantly, the NFL at low pressures changes to a Fermi liquid. Comparative phase diagrams [Fig. 1(c)] illustrate that the Mott-insulating QSL phase in $\kappa-\mathrm{Cu}_{2}(\mathrm{CN})_{3}$ is replaced by the NFL in $\kappa-\mathrm{HgBr}[37,42,43]$. Remarkably, $T_{c}$ forms a dome around the NFL-FL crossover pressure $[37,40]$. In the present work, we investigate the pressure evolution of the nature of electron pairing that occurs in a doped QSL candidate $\kappa$ - $\mathrm{HgBr}$ by electron transport and Nernst-effect measurements. The results demonstrate the pressure-tuned BEC-BCS crossover and clarify its relation to the Fermi liquidity above $T_{c}$.

\section{RESULTS AND DISCUSSION}

\section{A. Crossover from non-Fermi liquid to Fermi liquid}

To precisely associate the nature of electron pairing with that of the normal-state fluidity above $T_{c}$, we performed resistivity measurements under finely incremented pressures [25]. To avoid resistivity jumps due to microcracking of the crystal encountered in the ambient-pressure measurements, we had to apply finite pressures to obtain jumpfree resistivity data. As seen in Fig. 2(a), the temperature dependence of the in-plane resistivity $\rho(T)$ varies systematically with pressure; the linear temperature dependence changes into superlinear dependences at elevated pressures. Fitting the form of $\rho_{n}(T)=\rho_{0}+A T^{\alpha}$ to the normal-state 
(a)
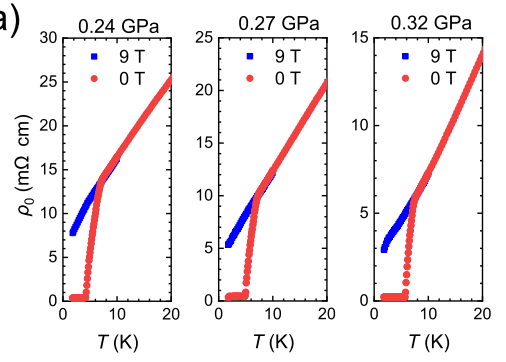

(b)

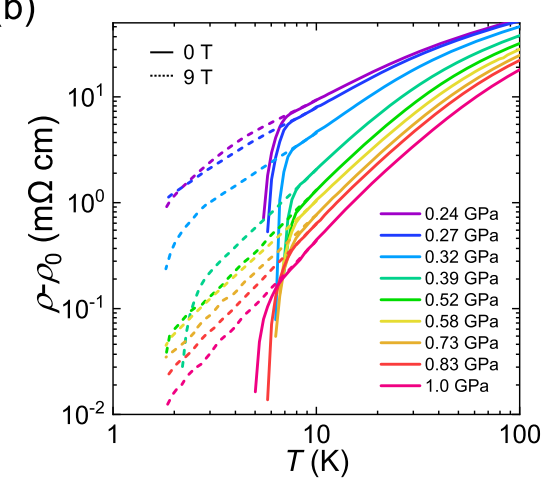

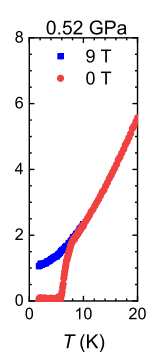
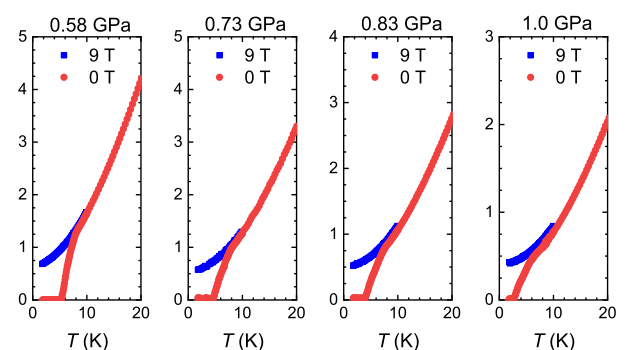

(c)

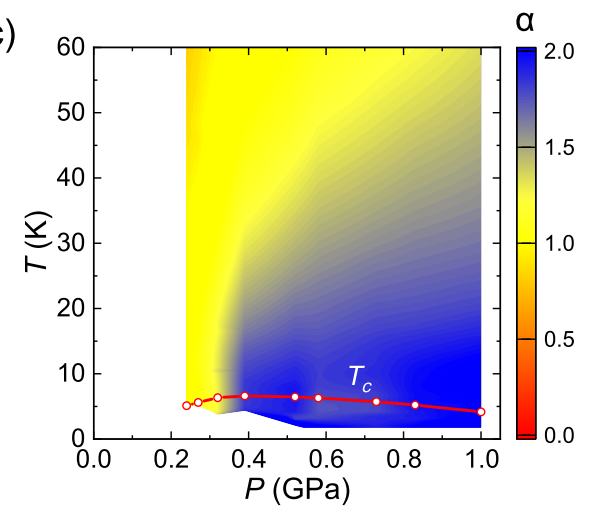

FIG. 2. Temperature dependence of resistivity under pressures. (a) Temperature variation of the in-plane resistivity $\rho(T)$ at $0 \mathrm{~T}$ and a perpendicular field of 9 T. (b) Logarithmic plot of the temperature-dependent part of resistivity, $\rho(T)-\rho_{0}$, with $\rho_{0}$ being the residual resistivity (see main text for its determination). The solid and dashed lines indicate the resistivity at $H=0$ and $9 \mathrm{~T}$, respectively. (c) Contour plot of the exponent $\alpha$ in fits of the form $A T^{\alpha}$ to the temperature-dependent part of the experimental in-plane resistivity $\rho(T)-\rho_{0} ; \alpha$ is determined by $\alpha(T) \equiv d \log \left[\rho(T)-\rho_{0}\right] / d \log T$. The yellow region $(\alpha \approx 1)$ corresponds to a non-Fermi-liquid phase, and the blue region $(\alpha \approx 2)$ corresponds to the Fermi-liquid (FL) phase.

resistivity $\rho(T)$ below $10 \mathrm{~K}$ measured under 9 T yields the value of the residual resistivity $\rho_{0}$ for each pressure. The logarithmic plot of $\left[\rho(T)-\rho_{0}\right]$ versus $T$ [Fig. 2(b)] highlights the pressure evolution of the temperature-dependent part of resistivity. The slopes of these curves, given by $\alpha(T) \equiv d \log \left[\rho(T)-\rho_{0}\right] / d \log T$, are defined as the exponent at temperature $T$. The contour plot of the deduced $\alpha(T)$ values on the pressure-temperature plane [Fig. 2(c)] shows that at low temperatures, $\alpha(T)$ is approximately unity before the pressure reaches $0.4 \mathrm{GPa}$ and sharply switches to the value of 2 at higher pressures, demonstrating that a NFL crosses over to a FL at approximately $0.35-0.40 \mathrm{GPa}$. This finding reproduces the previous results [37] and additionally reveals that the crossover is so sharp, as theoretically suggested [45], that it is reminiscent of a quantum phase transition [46-49]. This sharp crossover is regarded as a correspondence to the Mott transition in $\kappa-\mathrm{Cu}_{2}(\mathrm{CN})_{3}$, as implied by Fig. 1(c); at the crossover, the interaction strength crosses a Mott critical value at which the double occupancies of holes on a site, namely, Mottness, drastically changes [37], as theoretically suggested [38]. Furthermore, the NFL state does not only occur at the quantum critical point but extends to lower pressures, suggesting a stable quantum critical phase [50]. Such a NFL phase is also argued in a heavy electron system presumably hosting a QSL [51]. Charge carriers moving around in QSL phases may not achieve sufficient coherence, losing Fermi degeneracy and resulting in NFL phases. Theoretically, the temperature-linear resistivity observed in $\kappa-\mathrm{HgBr}$ at low pressures is suggested for bosonic charge excitations in a spin-charge separated doped spin liquid [17].

\section{B. Upper critical field and superconducting coherence length: BEC-BCS crossover}

Next, we explore how the nature of the superconducting condensate changes upon crossover from the anomalous metal to the ordinary metal by pressure. To assess the BEC or BCS nature, we evaluate the in-plane Ginzburg-Landau coherence length $\xi_{\|}$, which measures the Cooper-pair size, albeit not strictly in the BEC regime [52], from the perpendicular upper critical field $H_{c 2 \perp}$. To determine $H_{c 2 \perp}$, we traced the in-plane resistivity upon sweeping the magnetic field normal to the conducting layers shown at fixed temperatures [25]. Figure 3(a) shows the resistivity evolution upon the destruction of superconductivity by magnetic field at $0.32 \mathrm{GPa}$ as an example (see Ref. [25] for the resistivity data at other pressures: $0.24,0.27,0.32,0.39$, $0.52,0.58,0.73,0.83$, and $1.0 \mathrm{GPa})$. As the normal state shows no appreciable magnetoresistance as seen in the figure, the field dependence of the resistivity stems from the mobile vortices and/or superconducting fluctuations. The resistivity behavior in the magnetic-field-temperature 

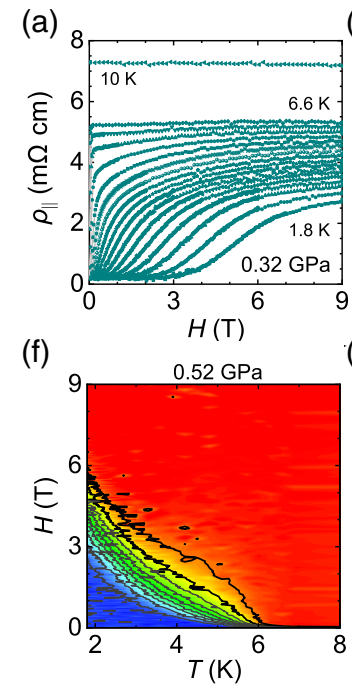

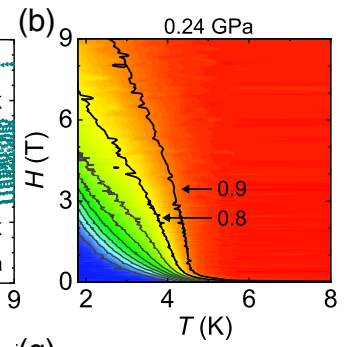

(g)

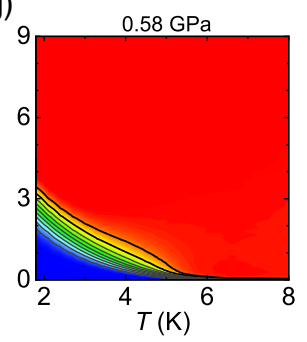

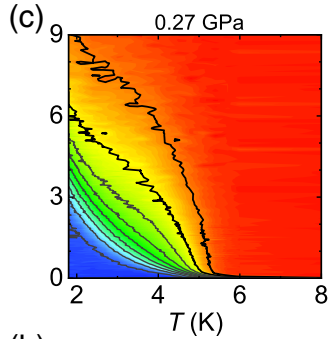

(h)

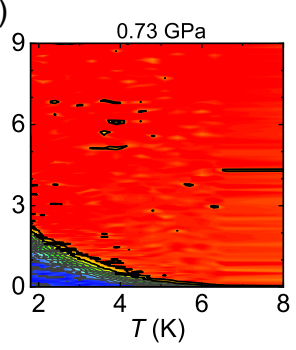

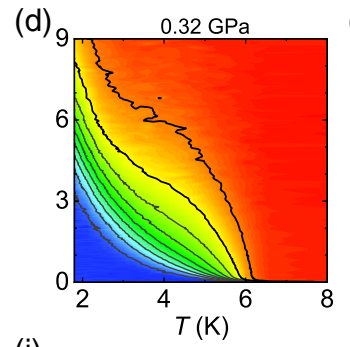

(i)

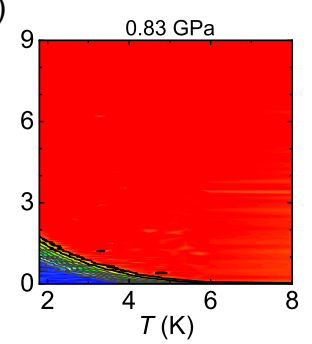

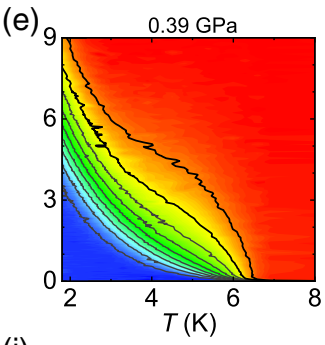

(j)

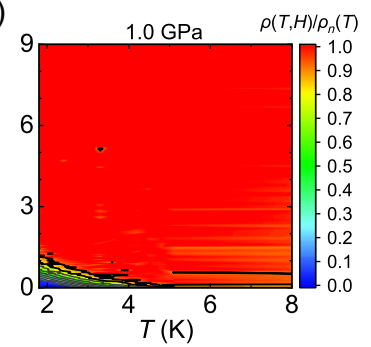

FIG. 3. Resistivity under magnetic field. (a) In-plane resistivity as a function of magnetic field $H$ applied perpendicular to the layers under the pressure of $0.32 \mathrm{GPa}$. The temperature was set to the temperatures between 1.8 and $6.6 \mathrm{~K}$ at $0.3 \mathrm{~K}$ intervals and to $10.0 \mathrm{~K}$. (b)-(j) Contour plots of the normalized in-plane resistivity, $\rho(T, H) / \rho_{n}(T)$, in the temperature-magnetic-field $(T-H)$ plane for the pressure of $0.24,0.27,0.32,0.39,0.52,0.58,0.73,0.83$, and $1.0 \mathrm{GPa}$, where $\rho(T, H)$ is the in-plane resistivity at $T$ and $H$ and $\rho_{n}(T)$ is the normal resistivity with the form of $\left(\rho_{0}+A T^{\alpha}\right)$ fitting the high-field data (see text). The solid lines indicate the contours of $\rho(T, H) / \rho_{n}(T)=0.1,0.2,0.3,0.4,0.5,0.6,0.7,0.8$, and 0.9 .

$(H-T)$ plane at each pressure is shown in the contour plot of the reduced resistivity $\rho(T, H) / \rho_{n}(T)$, where $\rho_{n}(T)$ is the normal-state resistivity determined in Figs. 3(b)-3(j). In the blue area, the resistivity vanishes or is small. As the magnetic field increases, the system enters the mixed state with mobile vortices, $\rho(T, H) / \rho_{n}(T)$ takes finite values (light blue to yellow area), and eventually, the normal-state resistivity is restored, i.e., $\rho(T, H) / \rho_{n}(T) \approx 1$ (red area). In the low-pressure regime, there is a wide region with finite values in $\rho(T, H) / \rho_{n}(T)$, indicating that mobile vortices or superconducting fluctuations are developed even at high fields. In contrast, at high pressures, the superconductivity is fully suppressed by low magnetic fields, and the transition to the normal state is sharp, as in conventional superconductors. Overall, superconductivity is robust to magnetic fields at low pressures.

The coherence length $\xi_{\|}$is evaluated from the temperature derivative of $H_{c 2 \perp}$ near $T_{c}$ through $T_{c}\left[d H_{c 2 \perp}(T) /\right.$ $d T]_{T=T_{c}}=\phi_{0} / 2 \pi \xi_{\|}^{2}$, where $\phi_{0}$ is the flux quantum. In highly two-dimensional layered superconductors, finite resistivity is caused by vortex flow under perpendicular magnetic fields, as observed in this system [53]; therefore, the definition of $H_{c 2 \perp}$ is not straightforward. In such a case, $H_{c 2 \perp}$ is better characterized by an initial drop in resistivity. Here, we adopt as $H_{c 2 \perp}$ the $80 \%$ and $90 \%$ transition points $\left[\rho(T, H) / \rho_{n}(T)=0.8\right.$ and 0.9$]$, which are indicated by bold contours in Figs. 3(b)-3(j). The values of $\left[d H_{c 2 \perp}(T) / d T\right]_{T=T_{c}}$ for the $80 \%$ and $90 \%$ transition lines are shown in Fig. 4. Using these values, we determined the $\xi_{\|}$values, whose pressure dependence is also shown in Fig. 4. At pressures below $0.4 \mathrm{GPa}, \xi_{\|} \sim 3 \mathrm{~nm}$, whereas it increases steeply with pressure and reaches $\sim 20 \mathrm{~nm}$ at $1 \mathrm{GPa}$. Assuming a cylindrical Fermi surface, we evaluated the Fermi wave number $k_{F}$ from the Hall coefficient under pressure [25] and obtained the $k_{F} \xi_{\|}$value, an index of the BEC-BCS nature or, roughly speaking, the degree of

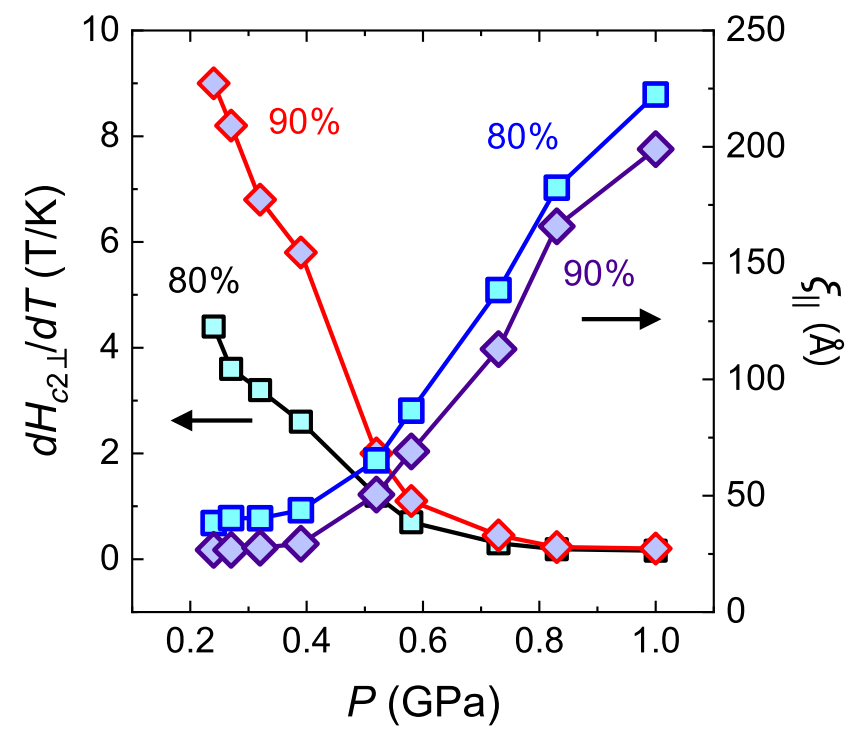

FIG. 4. Upper critical field and superconducting coherence length. The in-plane Ginzburg-Landau coherence length $\xi_{\|}$ calculated from the slope of the upper critical field, $d H_{c 2 \perp} / d T$ near $T_{c}$, where the $H_{c 2 \perp}$ is determined from the line at $\rho(T, H) / \rho_{n}(T)=0.8,0.9(80 \%$ and $90 \%$ of the normal-state resistivity) in Fig. 3. The squares and diamonds are the values determined for $\rho(T, H) / \rho_{n}(T)=0.8$ and 0.9 , respectively. 


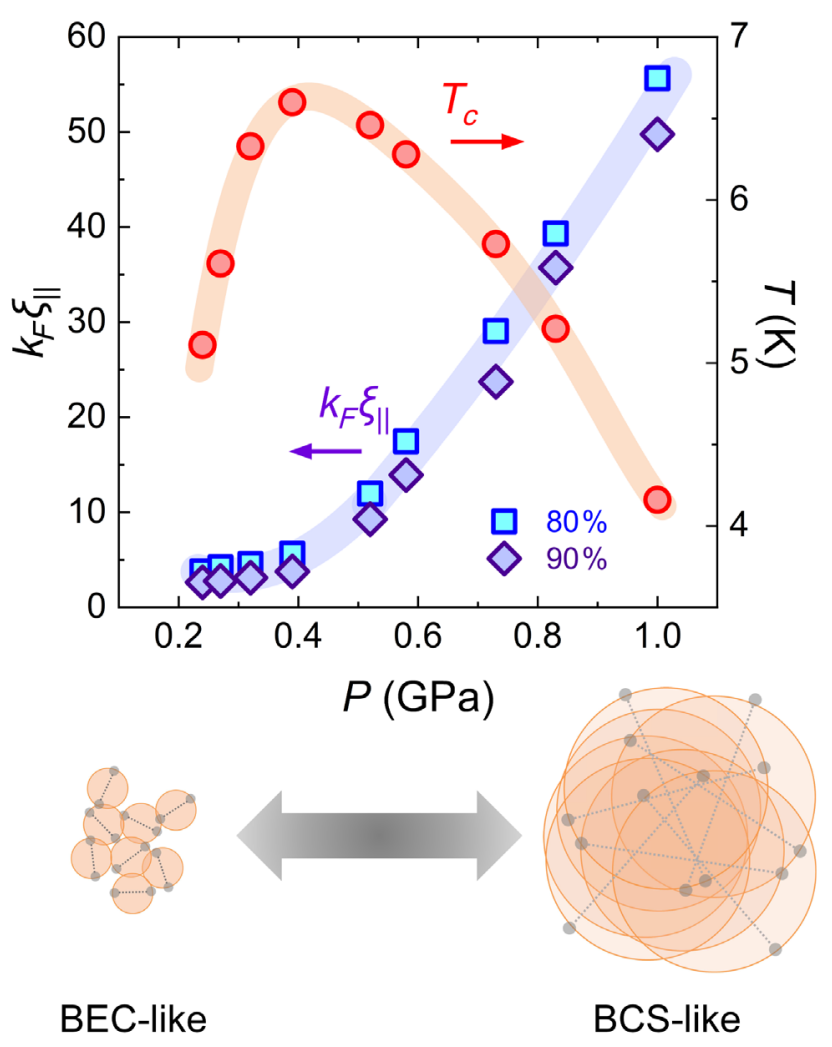

FIG. 5. Pressure dependence of $k_{F} \xi_{\|}$and BEC-BCS crossover. Superconducting transition temperature $T_{c}$ and $k_{F} \xi_{\|}$as a function of temperature, where $k_{F}$ is the Fermi wave number determined by the Hall coefficient [37]. The value of $k_{F} \xi_{\|}$is $\sim 3$ at low pressures and increases up to $\sim 50$ at $1 \mathrm{GPa}$ with pressure, pointing to a BEC-BCS crossover. Schematics drawn underneath the main panel depict the BEC- and BCS-like pairs on the lowand high-pressure sides.

overlap of Cooper pairs. The pressure dependence of $k_{F} \xi_{\|}$ is shown along with $T_{c}$ in Fig. 5. At high pressures, e.g., $1 \mathrm{GPa}, k_{F} \xi_{\|}$reaches $\sim 50$, pointing to highly overlapping pairs as in the conventional BCS regime, whereas at low pressures, $k_{F} \xi_{\|}$is as small as $\sim 3$; the size of the Cooper pair may become even smaller than the evaluated $\xi_{\|}$value when approaching the BEC regime according to a theoretical suggestion [52]. The $k_{F} \xi_{\|}$value of the order of unity signifies that the pairing is BEC-like, while the value of several tens points to BCS-like pairing, thus demonstrating that pressure induces a BEC-to-BCS crossover. The fieldrobust vortex liquid state and fluctuations at low pressures are consistent with the short coherence length and small density of the bosonic pairs inherent in BEC-like pairing, as shown theoretically [54].

\section{Nernst effect and superconducting fluctuations}

We further verified the preformed nature and magneticfield robustness of the pairing by examining the Nernst effect, in which a thermal flow generated by a temperature

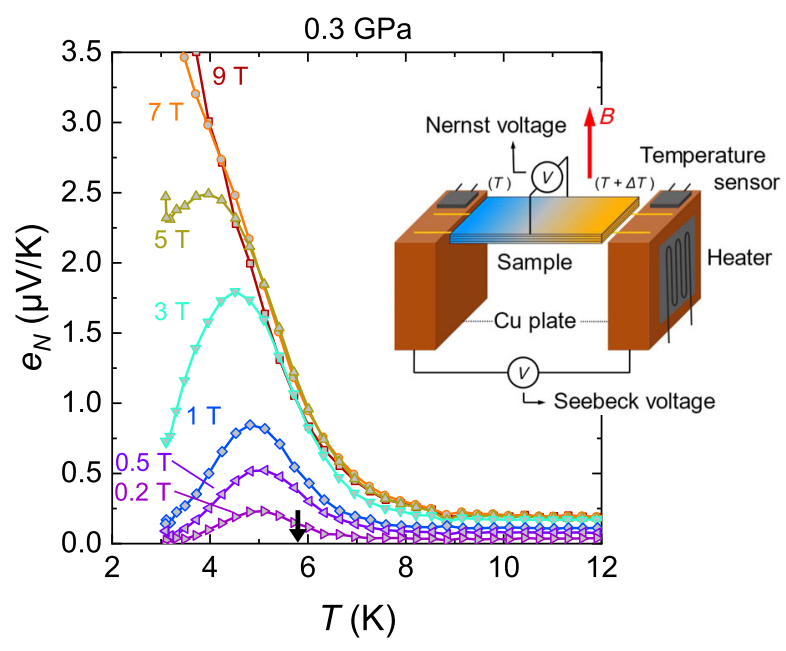

FIG. 6. Temperature dependence of the Nernst signal $e_{N}$ under a pressure of $0.30 \mathrm{GPa}$. The measurements were made at the magnetic fields of $0.2,0.5,1,3,5,7$, and $9 \mathrm{~T}$ applied perpendicular to the conducting layers. The arrow indicates the zero-field $T_{c}$ determined by the simultaneously measured thermopower. The geometry of the measurement setup is depicted in the inset.

gradient $\nabla_{x} T$ under a perpendicular magnetic field induces a transverse electric field $E_{y}$ (see the inset of Fig. 6). This effect, characterized by $e_{N} \equiv \pm E_{y} / \nabla_{x} T$ (with the sign depending on the field direction), is known to be enhanced by phase fluctuations arising from vortex liquid or preformed pairs in the superconducting state under magnetic fields, as observed in real materials such as cuprate and organic superconductors [10,55-59]. Figure 6 shows the temperature variation of $e_{N}$ at several fixed magnetic fields under a pressure of $0.3 \mathrm{GPa}$. The $e_{N}$ value, which is small in the normal state, starts to increase at temperatures well above $T_{c}$ upon cooling, signaling superconducting preformation; it is remarkable that $e_{N}$ shows a field-insensitive universal temperature variation at high magnetic fields. Figures 7(a)-7(d) display the magnetic-field variation of $e_{N}$ at several fixed temperatures under the pressures of $0.3,0.5$, 0.65 , and $0.9 \mathrm{GPa}$. It is evident that the magnetic-field profile of $e_{N}$ is strongly pressure dependent; the $e_{N}$ value remains large even at the maximum field, $9 \mathrm{~T}$, under $0.3 \mathrm{GPa}$, whereas under $0.9 \mathrm{GPa}$ it rapidly drops above $1 \mathrm{~T}$. To visualize the pressure evolution of the Nernst effect, we display the contour plots of $e_{N}$ in the temperaturemagnetic-field plane for each pressure in Figs. 7(e)-7(h). At $0.3 \mathrm{GPa}$, the ridge fields at which $e_{N}$ takes large values are extended vertically and even tend to go beyond the experimental maximum field, 9 T. In general, quasi-twodimensional superconductivity is easily suppressed by the perpendicular magnetic field far less than the Pauli limiting field, which is $\sim 10 \mathrm{~T}$ at $0.3 \mathrm{GPa}$, due to the orbital depairing. No such symptom up to $9 \mathrm{~T}$ and higher evidences unusual robustness of the pairing to the magnetic field, in line with the view of the BEC condensate. 


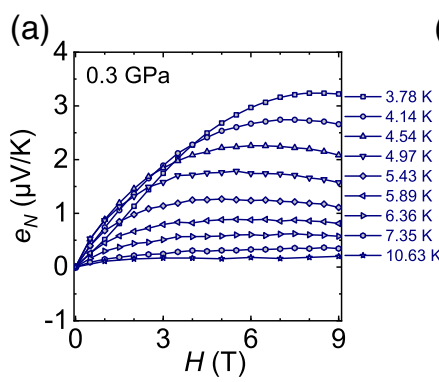

(e)

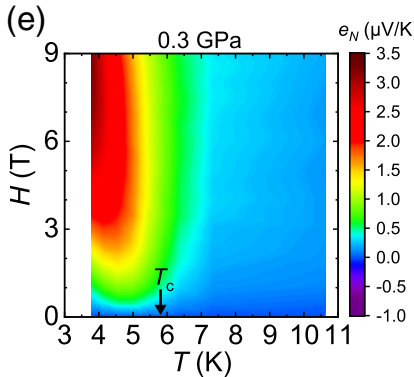

(b)

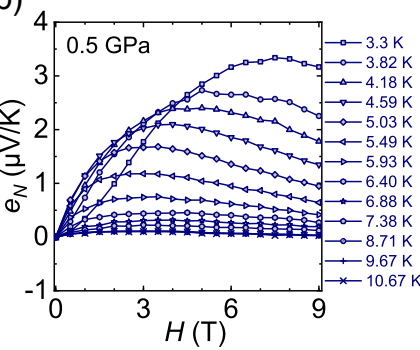

(f)

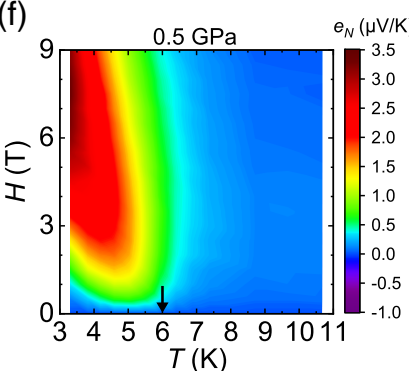

(c)

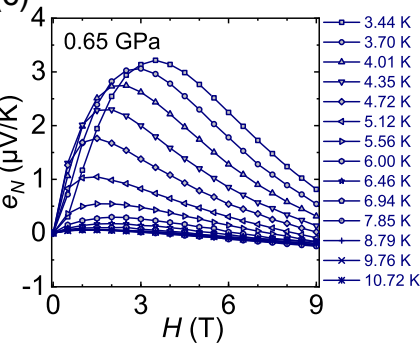

(d)

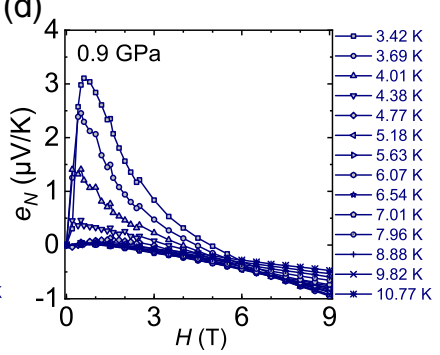

(g)

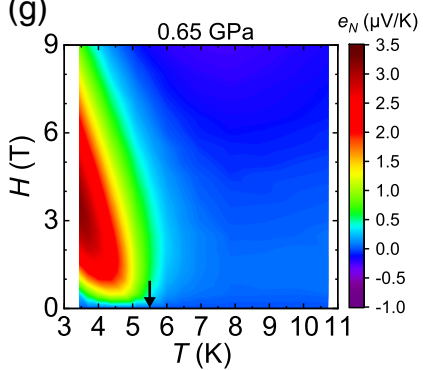

(h)

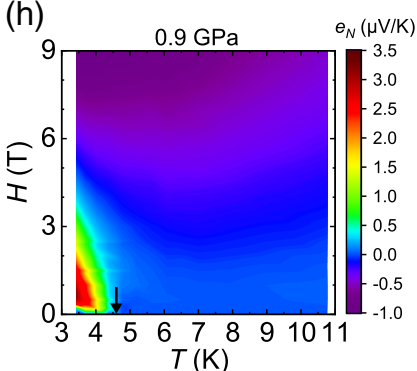

FIG. 7. Magnetic-field-temperature profile of the Nernst signal $e_{N}$ under different pressures. (a)-(d) Nernst signal $e_{N}$ traced upon sweeping the perpendicular magnetic field at several fixed temperatures under the pressures studied: $0.3,0.50 .65$, and $0.9 \mathrm{GPa}$. (e)-(h) Contour plots of the Nernst signal $e_{N}$ in the temperature-magnetic-field plane at the pressures of 0.3, 0.5 0.65, and 0.9 GPa. The red areas indicate hot Nernst regions. The arrows indicate the zero-field $T_{c}$.

At higher pressures, the ridge fields become suppressed along with $T_{c}$ and inclined, as in conventional superconductors. To see the pressure-temperature profile of $e_{N}$, we make a contour plot of the $T_{c}$ values at the maximum experimental field, $9 \mathrm{~T}$ (Fig. 8), which illustrates that the field-robust Nernst signal and its persistence at high temperatures far beyond $T_{c}$ is specific to the low-pressure phase. This is the Nernst behavior typical of BEC-like condensates; the effect of preformed pairs is expected to set

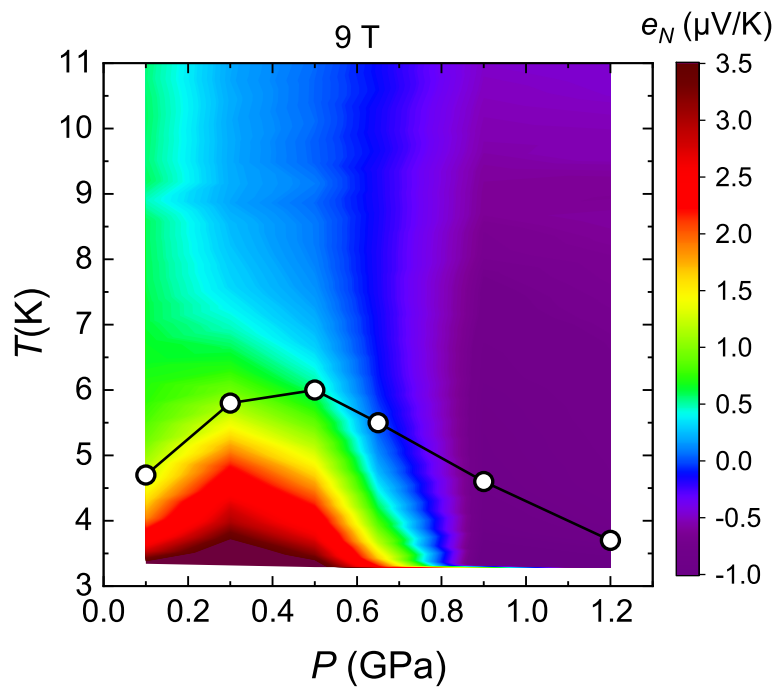

FIG. 8. Temperature-pressure profile of the Nernst signal $e_{N}$. The contour plot of the Nernst signal $e_{N}$ at a field of $9 \mathrm{~T}$ is shown in the temperature-pressure plane. The white circles indicate the zero-field $T_{c}$. in at higher temperatures in the Nernst signal than in the resistivity [10], consistent with the present observations. The systematic change of the Nernst profile with pressure lends support to the BEC-BCS crossover of the superconducting condensate.

It is reported that the ${ }^{13} \mathrm{C}$ NMR relaxation rate divided by temperature, $1 / T_{1} T$, shows a decrease below 7-9 K [60] despite that $T_{c} \sim 4.2 \mathrm{~K}$ at ambient pressure and the decrease becomes less prominent at higher pressures. This behavior is a likely manifestation of the preformed pairs in the BEC-like regime at ambient pressure and its crossover to the BCS regime at high pressures. We also note that the recent torque measurements suggest the fluctuating superconductivity that sets in at around $7 \mathrm{~K}$ at ambient pressure, implying the preformation of the Cooper pairs [61].

\section{CONCLUDING REMARKS}

The electron transport and Nernst effect in the doped triangular system, $\kappa-\mathrm{HgBr}$, revealed that the nature of the superconductivity crosses over from BEC-like to BCS pairing with increasing pressure. The present BEC-BCS crossover is associated with a NFL-FL crossover, at which the Coulombic repulsive energy relative to the band width exceeds a critical value for a Mott transition that would occur unless doped. To explore the nature of fermionic pairing that encompasses the BEC and BCS regimes, it is crucial to vary the control parameter linked to the interparticle interaction across some critical value at which the nature of the interaction dramatically changes. In cold 
fermionic atoms, the controlling parameter is the magnetic field, which varies interatomic interactions across a critical value through the Feshbach resonance. In the present case of interacting electrons, pressure is a parameter that varies the Coulombic interactions across the Mott critical value at which the double occupancies of carriers are critically allowed or forbidden [38]. This Mott-driven BEC-BCS crossover is a novel addition to the physics of the pairing instability of interacting fermions.

\section{ACKNOWLEDGMENTS}

We thank M. Ogata for fruitful discussions. This work was supported by Japan Society for the Promotion of Science (Grants No. 18H05225, No. 19H01846, No. 20K20894, and No. 20KK0060). Several experiments were performed using facilities of the Cryogenic Research Center, the University of Tokyo.

\section{APPENDIX: METHODS}

\section{Measurements of resistivity}

The in-plane resistivity was measured by the conventional four-probe method; four gold wires were attached on the planar surface of a crystal with carbon paste. The Quantum Design physical property measurement system (PPMS) was used for the resistivity measurements. To investigate the perpendicular upper critical field $H_{c 2 \perp}$, we traced the resistivity with the magnetic field applied perpendicular to the conducting layers up to $9 \mathrm{~T}$. In organic conductors, the rapid cooling often causes unwanted crystal cracking and/or conformational disorder of terminal ethylene groups in BEDT-TTF. To avoid suffering from these, the cooling rate was kept below $0.5 \mathrm{~K} / \mathrm{min}$.

\section{Measurements of the Nernst effect}

The Nernst voltage $e_{N}$ was generated in a direction perpendicular to both the directions of an externally created temperature gradient and an applied magnetic field (see the inset of Fig. 6). The magnetic field was applied perpendicular to the conducting layers. The temperature difference across the crystal $\Delta T$ along the two-dimensional plane was kept less than $0.5 \mathrm{~K}$ in the field-sweep measurements and less than $T / 10$ in the temperature-sweep measurements. The temperatures were measured with the Cernox sensors (Lake Shore Cryotronics, Inc.) attached on the cold and hot side of the crystal. The Nernst-effect measurements were performed under several pressures and the Cernox sensors were calibrated every time pressure was changed, using a reference thermometer. To determine the Nernst voltage $e_{N}$, the transverse voltage $V$ was measured under both positive and negative fields $(+H$ and $-H)$, and then the $e_{N}$ was obtained by antisymmetrizing the two voltages by $e_{N}(H)=[V(+H)-V(-H)] / 2$. Through this measurement, PPMS was used as a controller of magnetic field and temperature; the temperature gradient in the sample was generated by a heater attached on one side of the crystal and the sample temperature was defined by the mean value of the temperatures of the hot and cold sides. As in the resistivity measurements, the cooling rate was kept below $0.5 \mathrm{~K} / \mathrm{min}$. The thermopower was also measured along with the Nernst voltage for the identical sample in order to know the $T_{c}$ of the sample used.

\section{Pressurization}

To apply hydrostatic pressures to the sample, a dual structured clamp-type pressure cell made from $\mathrm{BeCu}$ and $\mathrm{NiCrAl}$ cylinders was used with the Daphne 7373 oil as a pressure-transmitting medium. The pressure values quoted in this article are the internal pressures at low temperatures determined with the manganin pressure gauge or the tin manometer. According to Ref. [62], the clumped pressure gradually decreases by 1.5-2.0 kbar on cooling from 300 to $50 \mathrm{~K}$ and is nearly constant below that.

[1] M. Imada, A. Fujimori, and Y. Tokura, Metal-Insulator Transitions, Rev. Mod. Phys. 70, 1039 (1998).

[2] J. Bardeen, L. N. Cooper, and J. R. Schrieffer, Theory of Superconductivity, Phys. Rev. 108, 1175 (1957).

[3] V. J. Emery and S. A. Kivelson, Importance of Phase Fluctuations in Superconductors with Small Superfluid Density, Nature (London) 374, 434 (1995).

[4] Y. J. Uemura, L. P. Le, G. M. Luke, B. J. Sternlieb, W. D. Wu, J. H. Brewer, T. M. Riseman, C. L. Seaman, M. B. Maple, M. Ishikawa, D. G. Hinks, J. D. Jorgensen, G. Saito, and H. Yamochi, Basic Similarities among Cuprate, Bismuthate, Organic, Chevrel-Phase, and Heavy-Fermion Superconductors Shown by Penetrationdepth Measurements, Phys. Rev. Lett. 66, 2665 (1991).

[5] S. Kasahara, T. Watashige, T. Hanaguri, Y. Kohsaka, T. Yamashita, Y. Shimoyama, Y. Mizukami, R. Endo, H. Ikeda, K. Aoyama, T. Terashima, S. Uji, T. Wolf, H. von Löhneysen, T. Shibauchi, and Y. Matsuda, Field-Induced Superconducting Phase of FeSe in the BCS-BEC Cross-Over, Proc. Natl. Acad. Sci. U.S.A. 111, 16309 (2014).

[6] T. Hashimoto, Y. Ota, A. Tsuzuki, T. Nagashima, A. Fukushima, S. Kasahara, Y. Matsuda, K. Matsuura, Y. Mizukami, T. Shibauchi, S. Shin, and K. Okazaki, BoseEinstein Condensation Superconductivity Induced by Disappearance of the Nematic State, Sci. Adv. 6, eabb9052 (2020).

[7] Y. Nakagawa, Y. Kasahara, T. Nomoto, R. Arita, T. Nojima, and Y. Iwasa, Gate-Controlled BCS-BEC Crossover in a Two-Dimensional Superconductor, Science 372, 190 (2021).

[8] C. A. Regal, M. Greiner, and D. S. Jin, Observation of Resonance Condensation of Fermionic Atom Pairs, Phys. Rev. Lett. 92, 040403 (2004).

[9] M. W. Zwierlein, C. A. Stan, C. H. Schunck, S. M. F. Raupach, A. J. Kerman, and W. Ketterle, Condensation of Pairs of Fermionic Atoms near a Feshbach Resonance, Phys. Rev. Lett. 92, 120403 (2004). 
[10] Q. Chen, J. Stajic, S. Tan, and K. Levin, BCS-BEC Crossover: From High Temperature Superconductors to Ultracold Superfluids, Phys. Rep. 412, 1 (2005).

[11] M. Randeria and E. Taylor, Crossover from BardeenCooper-Schrieffer to Bose-Einstein Condensation and the Unitary Fermi Gas, Annu. Rev. Condens. Matter Phys. 5, 209 (2014).

[12] Y. Zhou, K. Kanoda, and T.-K. Ng, Quantum Spin Liquid States, Rev. Mod. Phys. 89, 025003 (2017).

[13] L. Savary and L. Balents, Quantum Spin Liquids: A Review, Rep. Prog. Phys. 80, 016502 (2017).

[14] C. Broholm, R. J. Cava, S. A. Kivelson, D. G. Nocera, M. R. Norman, and T. Senthil, Quantum Spin Liquids, Science 367, eaay0668 (2020).

[15] P. W. Anderson, The Resonating Valence Bond State in $\mathrm{La}_{2} \mathrm{CuO}_{4}$ and Superconductivity, Science 235, 1196 (1987).

[16] M. Ogata, Superconducting States in Frustrating t-J model: A Model Connecting High- $T_{c}$ Cuprates, Organic Conductors and $\mathrm{Na}_{x} \mathrm{CoO}_{2}$, J. Phys. Soc. Jpn. 72, 1839 (2003).

[17] P. A. Lee, N. Nagaosa, and X.-G. Wen, Doping a Mott Insulator: Physics of High-Temperature Superconductivity, Rev. Mod. Phys. 78, 17 (2006).

[18] P. A. Lee, From High Temperature Superconductivity to Quantum Spin Liquid: Progress in Strong Correlation Physics, Rep. Prog. Phys. 71, 012501 (2008).

[19] J. Zaanen and B. J. Overbosch, Mottness Collapse and Statistical Quantum Criticality, Phil. Trans. R. Soc. A 369, 1599 (2011).

[20] B. J. Powell and R. H. McKenzie, Quantum Frustration in Organic Mott Insulators: From Spin Liquids to Unconventional Superconductors, Rep. Prog. Phys. 74, 056501 (2011).

[21] H. Yokoyama, M. Ogata, Y. Tanaka, K. Kobayashi, and H. Tsuchiura, Crossover between BCS Superconductor and Doped Mott Insulator of $d$-Wave Pairing State in TwoDimensional Hubbard Model, J. Phys. Soc. Jpn. 82, 014707 (2013).

[22] E. Gull, O. Parcollet, and A. J. Millis, Superconductivity and the Pseudogap in the Two-Dimensional Hubbard Model, Phys. Rev. Lett. 110, 216405 (2013).

[23] H.-C. Jiang, Superconductivity in the Doped Quantum Spin Liquid on the Triangular Lattice, npj Quantum Mater. 6, 71 (2021).

[24] R. N. Lyubovskaya, E. A. Zhilyaeva, A. V. Zvarykina, V. N. Laukhin, R. B. Lyubovskii, and S. I. Pesotskii, Is the Organic Metal (ET) ${ }_{4} \mathrm{Hg}_{3} \mathrm{Br}_{8}$ a Quasi-2D Superconductor?, JETP Lett. 45, 530 (1987).

[25] See Supplemental Material at http://link.aps.org/ supplemental/10.1103/PhysRevX.12.011016, which includes Refs. [26-33], for extended experimental data and supporting data analysis.

[26] R. Li, V. Petricek, G. Yang, P. Coppens, and M. Naughton, Room- and Low-Temperature Crystallographic Study of the Ambient Pressure Organic Superconductor (Bisethylene dithiotetrathiofulvalene) ${ }_{4} \mathrm{Hg}_{2.89} \mathrm{Br}_{8}, \quad$ Chem. Mater. 10, 1521 (1998).

[27] Y. Shimizu, M. Maesato, and G. Saito, Uniaxial Strain Effects on Mott and Superconducting Transitions in $\kappa-(\mathrm{ET})_{2} \mathrm{Cu}_{2}(\mathrm{CN})_{3}$, J. Phys. Soc. Jpn. 80, 074702 (2011).
[28] T. Komatsu, N. Matsukawa, T. Inoue, and G. Saito, Realization of Superconductivity at Ambient Pressure by Band-Filling Control in $\kappa-(\mathrm{ET})_{2} \mathrm{Cu}_{2}(\mathrm{CN})_{3}$, J. Phys. Soc. Jpn. 65, 1340 (1996).

[29] H. C. Kandpal, I. Opahle, Y.-Z. Zhang, H. O. Jeschke, and R. Valenti, Revision of Model Parameters for $\kappa$-Type Charge Transfer Salts: An Ab Initio Study, Phys. Rev. Lett. 103, 067004 (2009).

[30] T. Koretsune and C. Hotta, Evaluating Model Parameters of the $\kappa$ - and $\beta^{\prime}$-Type Mott Insulating Organic Solids, Phys. Rev. B 89, 045102 (2014).

[31] R. N. Lyubovskaya, E. I. Zhilyaeva, S. I. Pesotskii, R. B. Lyubovskii, L. O. Atovmyan, O. A. D'yachenko, and T. G. Takhirov, Superconductivity of $(\mathrm{ET})_{4} \mathrm{Hg}_{2.89} \mathrm{Br}_{8}$ at Atmospheric Pressure and $T_{c}=4.3 \mathrm{~K}$ and the Critical-Field Anisotropy, JETP Lett. 46, 188 (1987).

[32] R. J. Gillespie, D. Brown, W. R. Datars, K. R. Morgan, Z. Tun, and P. K. Ummat, The Preparation and Structure of Chain and Sheet Mercury Compounds, Phil. Trans. R. Soc. A 314, 105 (1985).

[33] T. Yamamoto, M. Uruichi, K. Yamamoto, K. Yakushi, A. Kawamoto, and H. Taniguchi, Examination of the ChargeSensitive Vibrational Modes in Bis(ethylenedithio)tetrathiafulvalene, J. Phys. Chem. B 109, 15226 (2005).

[34] Y. Shimizu, K. Miyagawa, K. Kanoda, M. Maesato, and G. Saito, Spin Liquid State in an Organic Mott Insulator with a Triangular Lattice, Phys. Rev. Lett. 91, 107001 (2003).

[35] F. L. Pratt, P. J. Baker, S. J. Blundell, T. Lancaster, S. OhiraKawamura, C. Baines, Y. Shimizu, K. Kanoda, I. Watanabe, and G. Saito, Magnetic and Non-Magnetic Phases of a Quantum Spin Liquid, Nature (London) 471, 612 (2011).

[36] S. Yamashita, Y. Nakazawa, M. Oguni, Y. Oshima, H. Nojiri, Y Shimizu, K. Miyagawa, and K. Kanoda, Thermodynamic Properties of a Spin-1/2 Spin-Liquid State in a kappa-Type Organic Salt, Nat. Phys. 4, 459 (2008).

[37] H. Oike, K. Miyagawa, H. Taniguchi, and K. Kanoda, Pressure-Induced Mott Transition in an Organic Superconductor with a Finite Doping Level, Phys. Rev. Lett. 114, 067002 (2015).

[38] C.-D. Hébert, P. Sémon, and A. M. S. Tremblay, Superconducting Dome in Doped Quasi-2D Organic Mott Insulators: A Paradigm for Strongly-Correlated Superconductivity, Phys. Rev. B 92, 195112 (2015).

[39] H. Oike, Y. Suzuki, H. Taniguchi, Y. Seki, K. Miyagawa, and K. Kanoda, Anomalous Metallic Behaviour in the Doped Spin Liquid Candidate $\kappa-(\mathrm{ET})_{4} \mathrm{Hg}_{2.89} \mathrm{Br}_{8}$, Nat. Commun. 8, 756 (2017).

[40] H. Taniguchi, T. Okuhata, T. Nagai, K. Satoh, N. Mori, Y. Shimizu, M. Hedo, and Y. Uwatoko, Anomalous Pressure Dependence of Superconductivity in Layered Organic Conductor, $\kappa$-(BEDT-TTF $)_{4} \mathrm{Hg}_{2.89} \mathrm{Br}_{8}$, J. Phys. Soc. Jpn. 76, 113709 (2007).

[41] B. Miksch, A. Pustogow, M. J. Rahim, A. A. Bardin, K. Kanoda, J. A. Schlueter, R. Hübner, M. Scheffler, and M. Dressel, Gapped Magnetic Ground State in Quantum Spin Liquid Candidate $\kappa$-(BEDT-TTF $)_{2} \mathrm{Cu}_{2}(\mathrm{CN})_{3}$, Science 372, 276 (2021).

[42] Y. Kurosaki, Y. Shimizu, K. Miyagawa, K. Kanoda, and G. Saito, Mott Transition from a Spin Liquid to a Fermi Liquid in 
the Spin-Frustrated Organic Conductor $\kappa-(\mathrm{ET})_{2} \mathrm{Cu}_{2}(\mathrm{CN})_{3}$, Phys. Rev. Lett. 95, 177001 (2005).

[43] T. Furukawa, K. Kobashi, Y. Kurosaki, K. Miyagawa, and K. Kanoda, Quasi-Continuous Transition from a Fermi Liquid to a Spin Liquid in $\kappa-(\mathrm{ET})_{2} \mathrm{Cu}_{2}(\mathrm{CN})_{3}$, Nat. Commun. 9, 307 (2018).

[44] F. Kagawa, K. Miyagawa, and K. Kanoda, Unconventional Critical Behaviour in a Quasi-Two-Dimensional Organic Conductor, Nature (London) 436, 534 (2005).

[45] L. F. Tocchio, H. Lee, H. O. Jeschke, R. Valentí, and C. Gros, Mott Correlated States in the Underdoped TwoDimensional Hubbard Model: Variational Monte Carlo versus a Dynamical Cluster Approximation, Phys. Rev. B 87, 045111 (2013).

[46] S. L. Sondhi, S. M. Girvin, J. P. Carini, and D. Shahar, Continuous Quantum Phase Transitions, Rev. Mod. Phys. 69, 315 (1997).

[47] S. Sachdev, Quantum Phase Transitions (Cambridge University Press, Cambridge, England, 1999).

[48] P. Gegenwart, Q. Si, and F. Steglich, Quantum Criticality in Heavy-Fermion Metals, Nat. Phys. 4, 186 (2008).

[49] T. Furukawa, K. Miyagawa, H. Taniguchi, R. Kato, and K. Kanoda, Quantum Criticality of Mott Transition in Organic Materials, Nat. Phys. 11, 221 (2015).

[50] S. Paschen and Q. Si, Quantum Phases Driven by Strong Correlations, Nat. Rev. Phys. 3, 9 (2021).

[51] H. Zhao, J. Zhang, M. Lyu, S. Bachus, Y. Tokiwa, P. Gegenwart, S. Zhang, J. Cheng, Y.-F. Yang, G. Chen, Y. Isikawa, Q. Si, F. Steglich, and P. Sun, Quantum-Critical Phase from Frustrated Magnetism in a Strongly Correlated Metal, Nat. Phys. 15, 1261 (2019).

[52] J. R. Engelbrecht, M. Randeria, and C. A. R. Sáde Melo, BCS to Bose Crossover: Broken-Symmetry State, Phys. Rev. B 55, 15153 (1997).

[53] M. Tinkham, Introduction to Superconductivity, 2nd ed. (Dover Publications, Mineola, NY, 2004).
[54] K. Adachi and R. Ikeda, Stabilization of the Vortex-Liquid State by Strong Pairing Interaction, Phys. Rev. B 99, 064508 (2019).

[55] Y. Wang, L. Li, and N. P. Ong, Nernst Effect in High-T Superconductors, Phys. Rev. B 73, 024510 (2006).

[56] B. L. Kang, M. Z. Shi, S. J. Li, H. H. Wang, Q. Zhang, D. Zhao, J. Li, D. W. Song, L. X. Zheng, L. P. Nie, T. Wu, and X. H. Chen, Preformed Cooper Pairs in Layered FeSeBased Superconductors, Phys. Rev. Lett. 125, 097003 (2020).

[57] M. S. Nam, A. Ardavan, S. J. Blundell, and J. A. Schlueter, Fluctuating Superconductivity in Organic Molecular Metals Close to the Mott Transition, Nature (London) 449, 584 (2007).

[58] M.-S. Nam, C. Mézière, P. Batail, L. Zorina, S. Simonov, and A. Ardavan, Superconducting Fluctuations in Organic Molecular Metals Enhanced by Mott Criticality, Sci. Rep. 3, 3390 (2013).

[59] K. Behnia and H. Aubin, Nernst Effect in Metals and Superconductors: A Review of Concepts and Experiments, Rep. Prog. Phys. 79, 046502 (2016).

[60] Y. Eto, M. Itaya, and A. Kawamoto, Non-FermiLiquid Behavior of the Organic Superconductor $\kappa$-(BEDT-TTF $)_{4} \mathrm{Hg}_{2.89} \mathrm{Br}_{8}$ Probed by ${ }^{13} \mathrm{C} N M R$, Phys. Rev. B 81, 212503 (2010).

[61] S. Imajo, S. Sugiura, H. Akutsu, Y. Kohama, T. Isono, T. Terashima, K. Kindo, S. Uji, and Y. Nakazawa, Extraordinary $\pi$-Electron Superconductivity Emerging from a Quantum Spin Liquid, Phys. Rev. Research 3, 033026 (2021).

[62] K. Yokogawa, K. Murata, H. Yoshino, and S. Aoyama, Solidification of High-Pressure Medium Daphne 7373, Jpn. J. Appl. Phys. 46, 3636 (2007). 